\title{
Bionomics and insecticide resistance of Aedes albopictus in Shandong, a high latitude and high-risk dengue transmission area in China
}

Hongmei Liu ${ }^{1,2^{*}}$, Luhong Liư ${ }^{3}$, Peng Cheng ${ }^{2}$, Linlin Yang ${ }^{3}$, Junhu Chen ${ }^{4}$, Yao Lu ${ }^{3}$, Haifang Wang ${ }^{2}$, Xiao-Guang Chen ${ }^{1^{*}}$ and Maoqing Gong ${ }^{2^{*}}$

\begin{abstract}
Background: Dengue fever outbreaks tend to spread northward in China, and Jining is the northernmost region where local dengue fever cases have been detected. Therefore, it is important to investigate the density of Aedes albopictus and its resistance to deltamethrin.

Methods: The Breteau index (BI) and container index (Cl) were calculated to assess the larval density of Ae. albopictus and human-baited double net trap (HDN) surveillance was performed in six subordinate counties (Rencheng, Yanzhou, Sishui, Liangshan, Zoucheng and Jiaxiang) of Jining City in 2017 and 2018. The resistance of Ae. albopictus adults to deltamethrin was evaluated using the World Health Organization (WHO) standard resistance bioassay. The mutations at Vgsc codons 1532 and 1534 were also analysed to determine the association between $\mathrm{kdr}$ mutations and phenotypic resistance in adult mosquitoes.

Results: The average BI, $\mathrm{Cl}$ and biting rate at Jining were 45.30, 16.02 and 1.97 (female /man/hour) in 2017 and 15.95, 7.86 and $0.59 \mathrm{f} / \mathrm{m} / \mathrm{h}$ in 2018, respectively. In August 26, 2017, when the first dengue fever case was diagnosed, the BI at Qianli village in Jiaxiang County was 107.27. The application of prevention and control measures by the government sharply decreased the BI to a value of 4.95 in September 3, 2017. The mortality of field-collected Ae. albopictus females from Jiaxiang was 41.98\%. 11532T, F1534L and F1534S mutations were found in domain III of the Vgsc gene. This study provides the first demonstration that both I1532T and F1534S mutations are positively correlated with the deltamethrin-resistant phenotype.
\end{abstract}

Conclusions: Mosquito density surveillance, resistance monitoring and risk assessment should be strengthened in areas at risk for dengue to ensure the sustainable control of Ae. albopictus and thus the prevention and control of dengue transmission.

Keywords: Aedes albopictus, Dengue fever, Bl, kdr, Surveillance

*Correspondence: liuhmm163@163.com; xgchen2001@hotmail.com; gmq2005@163.com

1 Department of Pathogen Biology, Guangdong Provincial Key Laboratory of Tropical Disease Research, School of Public Health, Southern Medical University, Guangzhou, People's Republic of China

${ }^{2}$ Shandong Institute of Parasitic Diseases, Shandong First Medical University \& Shandong Academy of Medical Sciences, Jining 272033, Shandong, People's Republic of China

Full list of author information is available at the end of the article

\section{Background}

Aedes albopictus, also known as the Asian tiger mosquito, can spread dengue fever [1], chikungunya fever [2] and Zika virus $[3,4]$ and has become one of the most invasive species in the world due to its strong ability to adapt to new environments [5]. China is undergoing rapid urbanization, which might lead to the emergence and spread of

c) The Author(s) 2020. This article is licensed under a Creative Commons Attribution 4.0 International License, which permits use, sharing, adaptation, distribution and reproduction in any medium or format, as long as you give appropriate credit to the original author(s) and the source, provide a link to the Creative Commons licence, and indicate if changes were made. The images or other third party material in this article are included in the article's Creative Commons licence, unless indicated otherwise in a credit line to the material. If material is not included in the article's Creative Commons licence and your intended use is not permitted by statutory regulation or exceeds the permitted use, you will need to obtain permission directly from the copyright holder. To view a copy of this licence, visit http://creativeco mmons.org/licenses/by/4.0/. The Creative Commons Public Domain Dedication waiver (http://creativecommons.org/publicdomain/ zero/1.0/) applies to the data made available in this article, unless otherwise stated in a credit line to the data. 
dengue fever due to urban population growth, crowded housing conditions and under-developed waste management systems [6].

In 2014, more than 40,000 cases of dengue fever, which is spread by Ae. albopictus, were reported in Guangzhou $[7,8]$, and the first local dengue fever case was reported in Hangzhou in 2015 [9] (Fig. 1). Shandong Province reported an autochthonous dengue case for the first time on 16 August 2017. Ninety-five cases were subsequently reported in the whole province, and 79 indigenous cases occurred in Jiaxiang, Jining City [10]. It is obvious that local cases of dengue tend to spread northward. The increase in dengue fever outbreaks in China observed in recent years is concerning. Unfortunately, considering the ability of Ae. albopictus to invade new habitats, the increasing frequency of dengue epidemics and the mobility of human populations, it is expected that new indigenous dengue fever cases will continue to occur in Shandong, China, which is similar to the situation in Brazil and some European regions, where dengue cases have emerged due to the introduction of dengue-infected people and invasion of Aedes mosquitoes [11, 12]. Dengue fever has become an important public health problem in China [13].
Mosquito surveillance has become an important component of integrated vector management programmes aiming to quantify human risk by determining the local vector abundance [14]. Mosquito surveillance mainly focuses on the collection of larvae and adult mosquitoes. The Breteau index (number of positive containers per 100 houses, BI) and the container index (percentage of water-holding containers infested with larvae or pupae, CI) $[15,16]$ are the most widely used larval indices for Aedes surveillance and the forecasting of disease outbreaks [17]. However, it should be noted that larval indices do not respond well to the abundance of adult mosquitoes. Adult sampling can provide more valuable data for the evaluation of dengue risk and adulticiding measures [16]. The human-baited double net trap (HDN) is an efficient method for Aedes monitoring. The HDN consists of two box nets; the inner net protects the human-bait, and the outer net is raised off the ground so that mosquitoes lured to the human-bait are collected between the nets by another collector, who is protected by repellent [18]. According to the Chinese vector monitoring programme [19], HDNs were mainly set to collect mosquitoes in the external environments near the

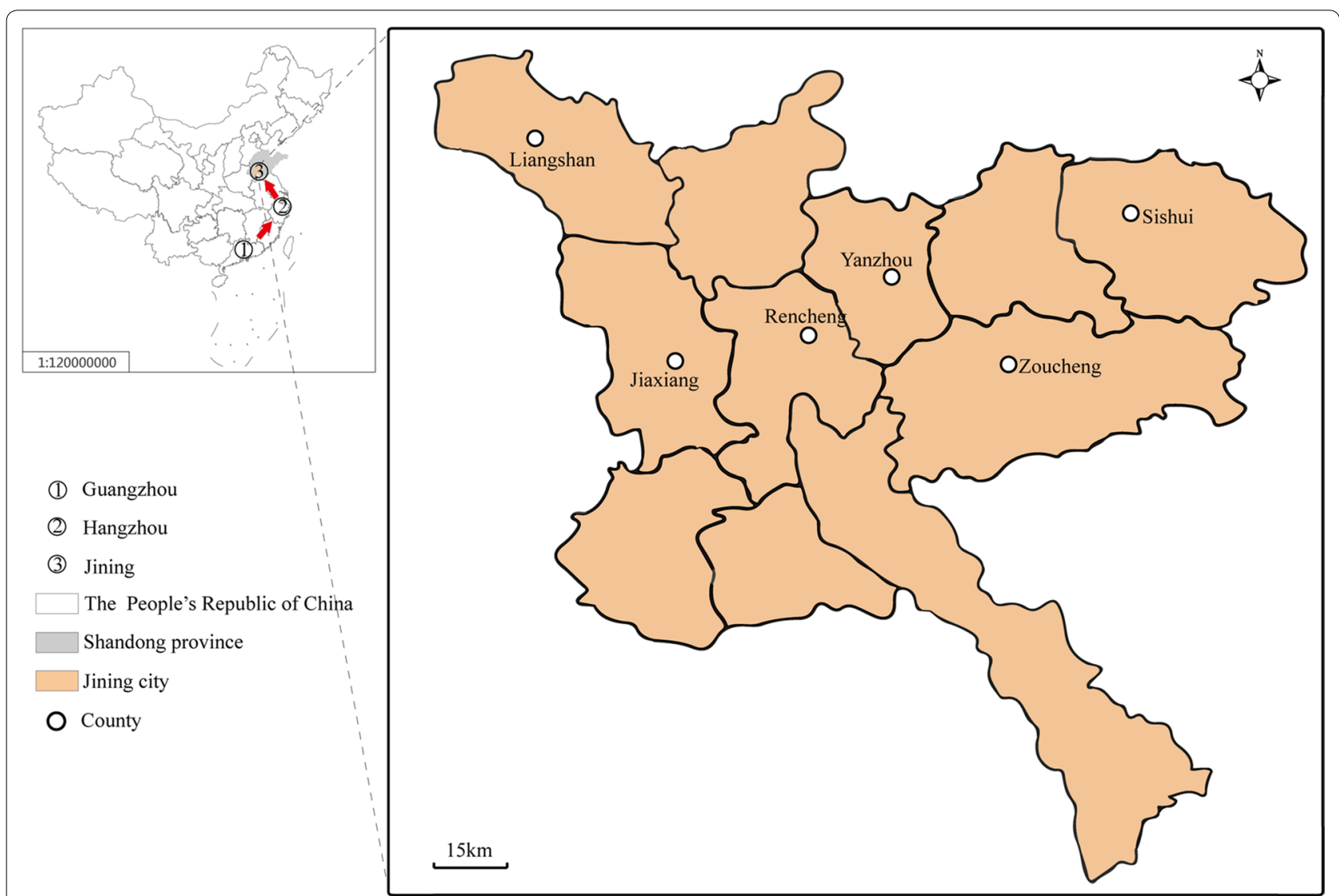

Fig. 1 Map of mosquito sample sites in Jining, China 
residential areas from $16: 30 \mathrm{~h}$ to $18: 30 \mathrm{~h}$ [18], and each collection was performed for a duration of $30 \mathrm{~min}$ [18]. According to the Chinese Center for Disease Control and Prevention's (CDC's) division standard for Aedes monitoring [20], a $\mathrm{BI}<5$ can be considered the threshold for controlling dengue fever and adult mosquito density by the HDN method should be less than 2. However, more evidence is needed to confirm that a $\mathrm{BI}<5$ is suitable for preventing dengue transmission because this value was derived from yellow fever transmission surveys [15, 21]. In this study, the larval density of Ae. albopictus was measured based on the $\mathrm{BI}$ and $\mathrm{CI}$, and adult mosquitoes were captured by the HDN method. Furthermore, the extent and intensity of pyrethroid resistance were evaluated through adult resistance surveillance and the assessment of knockdown resistance $(k d r)$ mutations.

\section{Methods}

\section{Study areas}

Shandong Province is located on the eastern coast of China, between $34^{\circ} 22^{\prime}, 38^{\circ} 24 \mathrm{~N}$ and $114^{\circ} 47^{\prime}, 122^{\circ} 42^{\prime} \mathrm{E}$. Shandong has a typical warm temperate climate with concentrated precipitation. In Shandong, rain and heat occur in the same season, and this area has short springs and autumns and long winters and summers. The annual average temperature is $11-14{ }^{\circ} \mathrm{C}$, and the annual average precipitation is between 550 and $950 \mathrm{~mm}$. However, the precipitation distribution is very uneven, with $60-70 \%$ of the annual precipitation concentrated in the summer, and this distribution is suitable for the development and reproduction of Ae. albopictus. Jining City, located in the southwestern part of Shandong Province, is dominated by plain depressions, has four national forest parks and is one of the central cities of the Huaihai Economic Zone.

According to the Chinese CDC's division standard for Aedes monitoring [20], Shandong Province can be classified as a class III region, which indicates that it is an area at risk for dengue fever outbreaks based on recently reported imported dengue cases and the distribution of Aedes mosquitoes. Six subordinate counties (Rencheng, Yanzhou, Sishui, Liangshan, Zoucheng and Jiaxiang) of Jining City were included as study areas in 2017 and 2018 (Fig. 1, Additional file 1: Table S1).

\section{Mosquito collection and species identification}

Mosquito surveillance was performed once a month in six subordinate counties (Rencheng, Yanzhou, Sishui, Liangshan, Zoucheng and Jiaxiang) of Jining City from June to September in 2017 and 2018. Each monitoring county selected no less than 100 households in four residential areas in different geographical locations. Other habitats, such as hospitals, parks, and waste collection stations, were subject to local conditions. All small water containers around each selected house were inspected for the presence of Aedes larvae or pupae, and the number of containers positive for Aedes mosquitoes was recorded. The larvae collected from the containers were identified to the species level or transported to the laboratory and reared to adulthood for species identification. All specimens were identified morphologically using taxonomic keys [22]. Molecular identification was conducted for selected adults and unhatched eggs using PCR with species-specific primers for amplification of the ribosomal internal transcribed spacers (ITS1 and ITS2) and $18 \mathrm{~S}$ rDNA regions [23-25].

Sampling was conducted twice a month from June to September in 2017 and 2018. The captured mosquitoes were killed by chloroform and then counted and identified based on morphology [26, 27].

Wild populations of Ae. albopictus were collected at Jiaxiang in August 2018. The larvae previously collected from the containers were transported to the laboratory and reared to adulthood under standard conditions of $27 \pm 2{ }^{\circ} \mathrm{C}$ and $75 \pm 10 \%$ relative humidity.

\section{Adult bioassay}

The larvae previously collected from the containers were transported to the laboratory and reared to adulthood. 3-5-days-old unfed female adults were tested using the World Health Organization (WHO) adult resistance bioassay.

According to WHO susceptibility testing guidelines [28], 20 to 25 female mosquitoes were exposed to $0.03 \%$ deltamethrin insecticide-impregnated papers (test) or carrier oil-treated papers without insecticide (control) for $1 \mathrm{~h}$ (School of Biological Sciences, Universiti Sains Malaysia, Penang, Malaysia) and then transferred to recovery tubes and maintained on $10 \%$ sucrose solution for $24 \mathrm{~h}$. The number of surviving and dead mosquitoes were recorded, and five replicates were included in the experiment. If the mosquito mortality in the control tubes exceeded $10 \%$, the mortality rates of all treated groups were corrected using Abbott's formula [28]. In the adult bioassay, the surviving mosquitoes were considered resistant, whereas the dead mosquitoes were considered susceptible [29]. The surviving and dead mosquitoes were separately stored in $95 \%$ ethanol for subsequent $k d r$ mutation genotyping.

\section{Genotyping of $k d r$ mutations}

Genomic DNA from individual mosquitoes was extracted using a DNeasy Blood and Tissue Kit (Qiagen, Hilden, Germany) following the manufacturer's recommended protocol. The molecular identification of Ae. albopictus was conducted using the species-specific primers aegSCF7 (5'-GAG AAC TCG CCG ATG AAC TT-3') and 
aegSCR7 (5'-GAC GAC GAA ATC GAA CAG GT-3) [30] to amplify the domain III region of the Ae. albopictus para-sodium channel gene $\alpha$ subunit. The PCR products were directly sequenced using Tks Gflex ${ }^{\mathrm{TM}}$ DNA Polymerase (Takara Bio, Inc., Shiga, Japan) following the manufacturer's recommended protocol. All resistant and susceptible individuals were genotyped to determine the presence of $k d r$ mutations at codons 1532 and 1534 by direct sequencing at Invitrogen Co., Ltd. (Shanghai, China).

\section{Data analysis}

The Ae. albopictus biting rate, which was used as the density indicator, was calculated as the mean number of adult female mosquito bites per man per hour $(\mathrm{f} / \mathrm{m} / \mathrm{h})$ $[1,31,32]$. Student's t-test was used to compare the mosquito density between 2017 and 2018 and the differences in the BIs and CIs. Heterogeneity of variance test assessed using Levene's test. The density of Ae. aegypti in different months and regions of the same year was compared with a one-way ANOVA followed by LSD tests (homogeneity of variance: $P>0.05$ ) or Dunnett's T3 tests (homogeneity of variance: $P<0.05$ ). The Ae. albopictus biting rates among the various months and regions were assessed using the Kruskal-Wallis test, and the $k d r$ allele frequencies of the resistant and susceptible groups were calculated. Fisher's exact tests were performed to assess the association between $k d r$ mutations and resistance, and the odds ratio for each $k d r$ allele was determined. The statistical analyses were performed using SPSS software (version 19 for Windows, SPSS Inc., Chicago, IL, USA).

According to the Aedes mosquito surveillance guidelines of China [20], a BI less than 5 is the threshold for the control of dengue transmission, whereas values of 5-10, $10-20$ and greater than 20 indicate risks of transmission, outbreak, and regional epidemic, respectively.
According to WHO guidelines [28], mortality rates in adult resistance bioassays of $98-100 \%, 90-97 \%$ and less than $90 \%$ indicate susceptible (S), 90-97\% suspected resistant (SR), and resistant (R) populations, respectively.

\section{Results}

\section{Breteau index}

The BI in Qianli village in Jiaxiang County was 107.27 in August 2017 (Fig. 2a, Additional file 2: Table S2). A sharp decrease in BI, was observed, reaching 4.95 in September 2017 (Fig. 2a, Additional file 2: Table S2).

The average BI values for Jining in 2017 and 2018 were 45.30 and 15.95, respectively. The BI value in 2017 was significantly higher than that in 2018 (t-test: $t_{(1426)}=$ 43.565, $P<0.001)$. The larval density showed seasonal fluctuations. The highest monthly average $\mathrm{BI}$ value for 2017 (BI = 66.88) was obtained in August, and the highest value for $2018(\mathrm{BI}=17.80)$ was recorded in August (Fig. 2a, Additional file 2: Table S2). During the survey, the highest BI value (107.27) in Jiaxiang was recorded in August 2017. Levene's test indicated that all the sample groups showed variance heterogeneity (all $P<0.05$ ). The differences in the $\mathrm{BI}$ among the various months were assessed by Welch's (unequal variance) ANOVA (2017: $F_{(3,538.657)}=164.627, P<0.001 ; 2018: F_{(3,208.989)}=61.778$, $P<0.001$ ) (Fig. 3a, Additional file 2: Table S2). It is worth noting that the BI values in August 2017 and September 2018 were higher than those in the other months of the same year, as determined by Dunnett's T3 test. In addition, a significant difference in BI values was found among the regions (Welch's ANOVA, 2017: $F_{(5,450.962)}=$ 156.172, $P<0.001$; 2018: $\left.F_{(5,120.669)}=969.668, P<0.001\right)$.

\section{Container index}

The average CI values of Jining in 2017 and 2018 were 16.02 and 7.86, respectively. The CI value for 2018 was clearly lower than that for 2017 (t-test: $t_{(1453)}=23.607$,
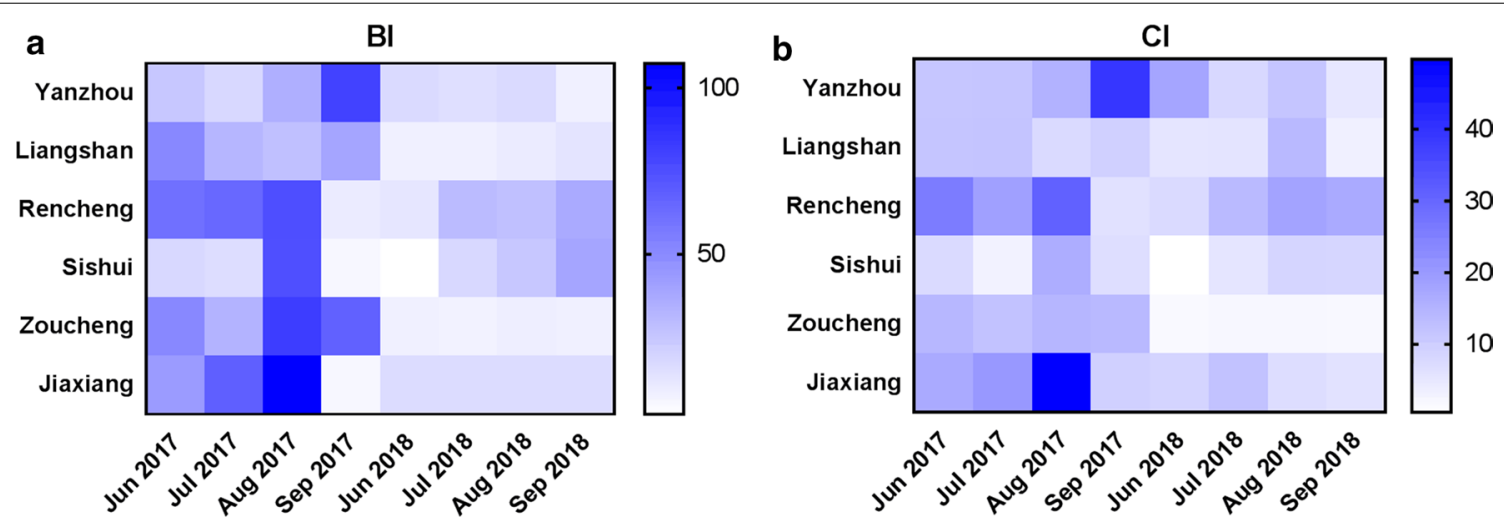

Fig. 2 The larval indices (BI and Cl) between 2017 and 2018. a The BI value between 2017 and 2018. b The Cl value between 2017 and 2018 

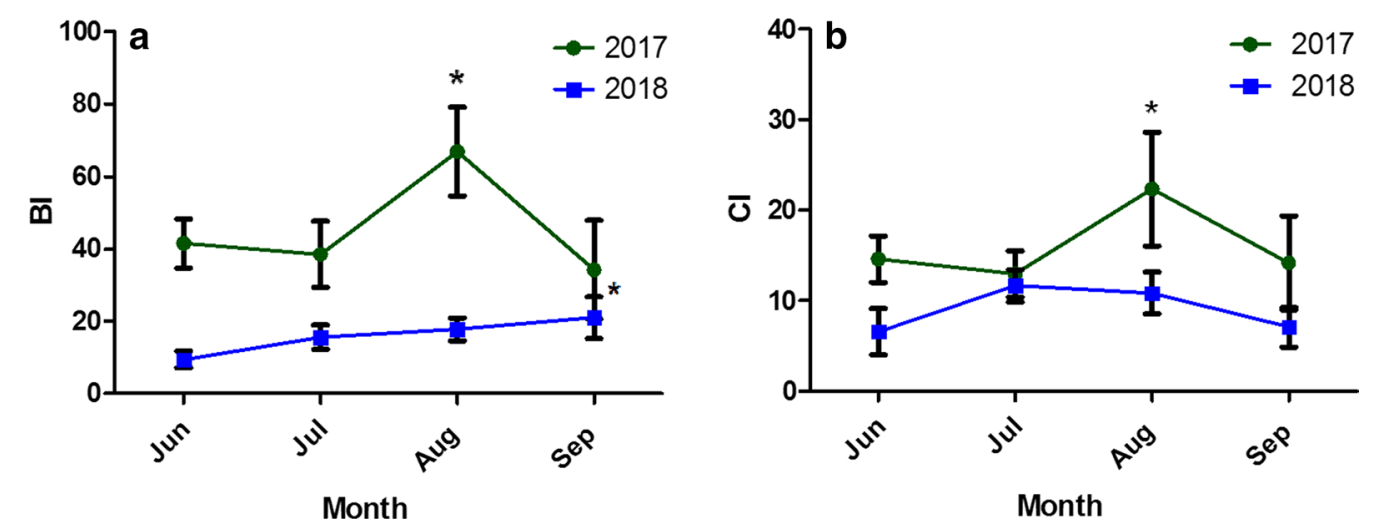

Fig. 3 Temporal variation of the larval indices ( $\mathrm{BI}$ and $\mathrm{Cl}$ ) between 2017 and 2018. a Trend in the monthly average BI fluctuation between 2017 and 2018. b Trend in the monthly average Cl fluctuation between 2017 and 2018

$P<0.001)$. The highest $\mathrm{CI}$ value (49.72) in Jiaxiang was also recorded in August 2017. The differences in the CI values among the various months were compared by Welch's ANOVA (2017: $F_{(3,202.691)}=44.276, P<0.001$; 2018: $\left.F_{(3,96.011)}=4.742, P=0.004\right)$. In 2017, the CI values in August and September were significantly different from other those in the months, whereas in 2018, the CI value in July was significantly different from that in August, as demonstrated by Dunnett's T3 test. A significant difference in the CI values was also found among the regions (Welch's ANOVA, 2017: $F_{(5,164.820)}=108.168$, $\left.P<0.001 ; 2018: F_{(5,76.931)}=304.864, P<0.001\right)$ (Figs. $2 \mathrm{~b}$, 3b, Additional file 3: Table S3).

\section{Human-baited double net trapping}

A total of 215 female Ae. albopictus were collected using the HDN technique. The average biting rates were 1.97 $\mathrm{f} / \mathrm{m} / \mathrm{h}$ in 2017 and $0.59 \mathrm{f} / \mathrm{m} / \mathrm{h}$ in 2018 (Fig. 4, Additional file 4: Table S4). In contrast, the number of collected specimens in 2017 was 3-fold higher than that in 2018, and the difference was statistically significant (t-test: $\left.t_{(49.37)}=6.946, P<0.001\right)$. In 2017, adult mosquitoes also showed a seasonal preference. The biting rates of $A e$. albopictus varied significantly among the various months (Kruskal-Wallis test, $X^{2}=23.721, d f=2, P<0.0001$ ) and regions (Kruskal-Wallis test, $\chi^{2}=27.850, d f=5, P<$ 0.0001 ) in 2017, but no significant differences in the biting rates were found among the various months (KruskalWallis test, $\left.\chi^{2}=5.466, d f=3, P=0.141\right)$ and regions (Kruskal-Wallis test, $\chi^{2}=8.596, d f=4, P=0.072$ ) in 2018.

\section{Adult bioassay and $k d r$ mutations}

The mortality of field-collected female Ae. albopictus from Jiaxiang, which is the northernmost location where local dengue fever has been detected in China, was

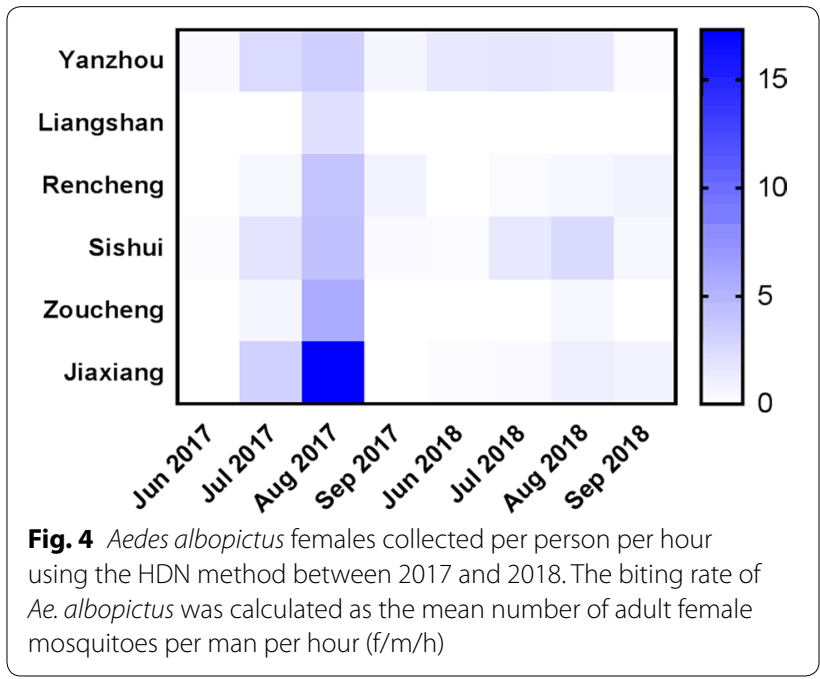

41.98\% (Table 1). Thus, according to the WHO guidelines, the mosquito population of Jiaxiang can be considered an R population. Forty-two susceptible (dead) and 60 resistant (surviving) individuals were reserved for the classification of resistance phenotypes.

Codons 1532 and 1534 in domain III of the Vgsc (voltage-gated sodium channel) gene exhibited non-synonymous mutations in 102 mosquito individuals from the Jiaxiang population. The wild-type allele ATC/I (93.63\%, $191 / 204)$ and the mutant allele ACC/T $(6.37 \%, 13 / 204)$ were detected at codon 1532, whereas the wild-type allele TTC/F (73.53\%, 150/204) and two mutant alleles, TTG/L (19.61\%, 40/204), TCC/S (6.86\%, 14/204), were detected at codon 1534. However, the mutant allele F1534C was not found in this population. The wild-type genotype I/I $(87.25 \%, 89 / 102)$ and the wild-type/mutant heterozygote I/T $(12.75 \%, 13 / 102)$ were detected at codon 1532 , but the mutant genotype $\mathrm{T} / \mathrm{T}$ was not detected. A total of six 
Table 1 Deltamethrin resistance of Ae. albopictus measured using the WHO standard adult bioassay

\begin{tabular}{llll}
\hline Sampling site & $n$ & Mortality \pm SE (\%) & Phenotype \\
\hline Jiaxiang & 102 & $41.98 \pm 13.79$ & $\mathrm{R}$ \\
Laboratory strain & 111 & $99.09 \pm 2.03$ & $\mathrm{~S}$
\end{tabular}

Abbreviations: $\mathrm{n}$, number of specimens; $\mathrm{R}$, resistant; $\mathrm{S}$, susceptible; $\mathrm{SE}$, standard error

genotypes, including the wild-type genotype F/F (55.88\%, $57 / 102)$, the wild-type/mutant heterozygotes $\mathrm{F} / \mathrm{L}$ (30.39\%, 31/102) and F/S (4.90\%, 5/102), and the mutant genotypes L/L (3.92\%, 4/102), S/S (3.92\%, 4/102) and L/S $(0.98 \%, 1 / 102)$, were detected at codon 1534 (Additional file 5: Table S5).

The I1532T mutant allele at codon 1532 was significantly correlated with deltamethrin resistance (odds ratio $=9.22, P=0.0084$ ) (Table 2 ). To assess the association between $k d r$ mutations at codon 1534 and resistance, the F1534S and F1534L alleles were analysed separately. The F1534S mutation was significantly associated with deltamethrin resistance (odds ratio $=4.56, P=0.0285$ ), but the F1534L mutation did not show significant differences between the $\mathrm{R}$ and $\mathrm{S}$ groups (odds ratio $=1.21$, $P=0.3663$ ) (Table 3).

\section{Discussion}

Dengue fever outbreaks tend to spread northward in China, and Jining is the northernmost region where local dengue fever cases had been observed. The present study constitutes the most comprehensive survey (by far) of
Ae. albopictus mosquitoes in Jining. This study found that the larval indices (BI and CI) and adult density in 2017 were obviously higher than those in 2018. The average BI values in Jining in 2017 and 2018 were 45.30 and 15.95, respectively, and these levels indicate potential risk of dengue outbreak. In addition, this study provides the first demonstration that both the I1532T and F1534S mutations are positively correlated with the deltamethrin resistance phenotype.

The BI is a key tool for determining the risk of dengue transmission [33] and is commonly used for risk assessment and as an early warning system for dengue epidemics $[15,17,34]$. In 2017, Jiaxiang, which had a BI value greater than 100 with reported dengue cases, was at risk of a regional epidemic. After the CDC administered health education programmes, destroyed mosquito populations, and optimized the environment, the BI value decreased sharply to 4.95 , and no new dengue fever cases were reported. This finding indicated that placing "anbei" into containers to kill larvae, spraying chemical insecticides, turning over containers, cleaning the water, and administering health education are effective measures for controlling dengue transmission, and these approaches were designed based on the experience in rural mosquito killing. Although the threshold used in this study was not statistically derived, the Chinese CDC states that a $\mathrm{BI}<5$ constitutes the threshold for the control of dengue transmission. Indeed, in this study, no new cases of dengue fever were recorded after the BI value decreased to less than 5; therefore, it appeared that a BI value $<5$ is a reliable threshold value capable of reflecting the incidence

Table 2 Association between $k d r$ mutations at codon 1532 and phenotypic resistance in Ae. albopictus

\begin{tabular}{|c|c|c|c|c|c|c|}
\hline \multirow{2}{*}{$\begin{array}{l}\text { Phenotype } \\
1532\end{array}$} & \multirow[t]{2}{*}{$n$} & \multicolumn{3}{|c|}{ Genotype } & \multirow[t]{2}{*}{ Odds ratio $(95 \% \mathrm{Cl})$} & \multirow[t]{2}{*}{$P$-value ${ }^{a}$} \\
\hline & & $\|$ & $\mathrm{IT}$ & $\mathrm{TT}$ & & \\
\hline$S$ & 42 & 41 & 1 & 0 & $9.22(1.18-72.36)$ & 0.0084 \\
\hline$R$ & 60 & 48 & 12 & 0 & & \\
\hline
\end{tabular}

a Fisher's exact probability test

Abbreviations: R, resistant; $\mathrm{S}$, susceptible; $\mathrm{n}$, number of samples; II, homozygous isoleucine/ isoleucine; IT, heterozygotes isoleucine/threonine; TT, homozygous threonine/threonine

Table 3 Association between kdr mutations at codon 1534 and phenotypic resistance in Ae. albopictus

\begin{tabular}{|c|c|c|c|c|c|c|c|c|c|c|c|}
\hline \multirow{2}{*}{$\begin{array}{l}\text { Phenotype } \\
1534\end{array}$} & \multirow[t]{2}{*}{$n$} & \multicolumn{6}{|c|}{ Genotype } & \multicolumn{2}{|c|}{ Odds ratio $(95 \% \mathrm{Cl})$} & \multicolumn{2}{|l|}{$P$-value ${ }^{a}$} \\
\hline & & $\mathrm{FF}$ & $\mathrm{FL}$ & $\mathrm{LL}$ & FS & LS & SS & F1534L & F1534S & F1534L & F1534S \\
\hline$S$ & 42 & 25 & 15 & 0 & 2 & 0 & 0 & $1.21(0.55-2.47)$ & $4.55(0.99-20.92)$ & 0.3663 & 0.0285 \\
\hline $\mathrm{R}$ & 60 & 32 & 16 & 4 & 3 & 1 & 4 & & & & \\
\hline
\end{tabular}

${ }^{a}$ Fisher's exact probability test

Abbreviations: $\mathrm{R}$, resistant; $\mathrm{S}$, susceptible; $\mathrm{n}$, number of samples; $\mathrm{FF}$, homozygous phenylalanine/phenylalanine; FL, heterozygotes phenylalanine/leucine, $\mathrm{LL}$, homozygous leucine/ leucine; LS, heterozygotes leucine/serine; SS, homozygous serine/serine 
of dengue epidemics and facilitating the management of dengue outbreaks. Related research has shown that BI could be used as a predictor of dengue transmission [15, 35], but the specific value of the low-risk BI threshold is controversial. Some studies have suggested that it should be $<1$ [15], whereas others have indicated that it should be $<4[36]$ or $<5[14,20]$. Thus, the low-risk BI threshold needs further study.

Notably, the BI values reached 44.75 and 15.95 in 2018 and 2017, respectively, and these levels represent risk of a regional epidemic and outbreak, respectively, but no dengue transmission was detected in any of the tested areas with the exception of Jiaxiang. This finding suggested that the outbreak of local dengue cases was not only closely related with Aedes mosquitoes but also with the infection source (dengue-infected people) and susceptible populations. This association might also be driven by a multifactorial process that includes lack of emphasis and recognition, environmental changes, urbanization and absence of talent. Jiaxiang has a large airport with frequent personnel exchanges and is adjacent to Nanyang Lake, and the New Zhuzhao River and Cai River pass through the district. Therefore, the Jiaxiang district is rich in water and rice in early July, which can increase the number of small-sized water bodies. The district has a large garage overflown with waste tires and used bottles. All of these features are suitable for mosquito breeding and could increase mosquito populations in the environment. Young adults who make a living in metropolitan and foreign countries during the slack seasons of farming and return during the busy seasons (June, July and August) might be infected with dengue and transport the virus to the area of study. The "left-behind" children and elderly individuals are more likely to show a lack of knowledge of dengue, which might result in a notable escalation of the likelihood of contact between humans and mosquitoes, and therefore, an increase in the risk of dengue transmission.

Due to the combination of risk factors exposed above, a new dengue case is expected to occur in Jiaxiang and even in Shandong Province. Therefore, strengthening surveillance, community mobilization and education could help reduce the incidence of dengue.

Because larvae do not directly transmit disease and the density of larvae does not represent the adult density, we also monitored the density of adult mosquitoes using the HDN method. This method exhibits a clear advantage in protecting attractors and remains an efficient monitoring method in epidemic areas and during dengue outbreaks [31, 37]. Similar research was performed in Hangzhou [32], where local dengue cases were first reported in August 2017 and the disease spread rapidly. The biting rate from September to October 2017 obtained using the HDN method was $2.29-8.50 \mathrm{f} / \mathrm{m} / \mathrm{h}$. A biting rate of $2-11 \mathrm{f} / \mathrm{m} / \mathrm{h}$ was obtained using the HDN method from October to November 2014 in Shenzhen [38], where an outbreak of dengue fever occurred in 2014. Previous studies have shown [11,33] that adult mosquito sampling can provide more valuable data for studies of seasonal population trends and for assessing the risk of dengue outbreaks compared with that obtained through larval sampling. However, the results obtained from adult sampling are less reproducible, and the density of Aedes mosquito larvae in different years is affected by natural and social factors during monitoring. Natural factors [35] include the geographical location, quantity and size of breeding sites, meteorological factors [39] and quantity of animals, whereas social factors [40, 41] mainly comprise the control activities implemented by governments and non-government organizations and the quality of health conditions. Therefore, the larval and adult mosquito density should be monitored simultaneously, and targeted prevention and control measures should be taken to effectively reduce the density of Aedes mosquitoes and the risk of dengue transmission.

Previous studies [29, 42-44] only detected non-synonymous mutations in domain III (1532 and 1534) in Ae. albopictus from China. Therefore, we focused on these two sites and found I1532T, F1534L and F1534S mutations. The results obtained in this study were similar to those obtained in other studies: the F1534S mutant allele greatly enhances the Vgsc insensitivity to deltamethrin, and the F1534L allele does not increase the resistance of mosquitoes to deltamethrin [29, 44, 45]. However, the other results showed differences. In the present study, the I1532T mutation, which appeared only in the mosquito populations from Italy and Hainan Province, China, was detected [45], and a previous study in Hainan showed that this mutation was negatively associated with the deltamethrin resistance phenotype [42]. However, in the present study, the I1532T mutation was positively correlated with the deltamethrin resistance phenotype. The F1534S and I1532T mutations reduce sensitivity to pyrethroids in mosquitoes, but the inactivation mechanism is unknown. $k d r$ mutations were found at other positions, including S989P and V1016G on Vgsc, in Aedes mosquitoes, and these mutations greatly increase the insensitivity to pyrethroids [30, 46-48]. In our study, the F1534C mutant allele, which is negatively correlated with pyrethroid resistance in southern China, was not found in this study area [49]. In addition, V1016G and V1016I mutation in IIS6 [29], which might be positively related to pyrethroid resistance, were not tested in our study. Thus, $k d r$ mutations need further research. 


\section{Conclusions}

Although the local circulation of dengue fever in China has not been clearly established [50], imported cases of dengue fever will continue to be reported for a long time, particularly in economically developed, border and densely populated areas. In addition, the risk of dengue fever has expanded to the north and west in China [51]. The geographical area and number of people at risk have increased significantly. In Jining and even Shandong Province, which constitutes a high-latitude area at high risk for dengue fever outbreak, Aedes density surveillance, resistance monitoring and risk assessment should be strengthened to ensure the sustainable control of Aedes and thus the prevention and control of dengue transmission.

\section{Supplementary information}

Supplementary information accompanies this paper at https://doi. org/10.1186/s13071-020-3880-2.

Additional file 1: Table S1. Mosquito sampling sites.

Additional file 2: Table S2. The BI value between 2017 and 2018.

Additional file 3: Table S3. The Cl value between 2017 and 2018.

Additional file 4: Table S4. Biting rate of Ae. albopictus females collected per person per hour using the HDN method.

Additional file 5: Table S5. Genotypes at codons 1532 and 1534 in $A e$ albopictus.

\section{Abbreviations}

Vgsc: voltage-gated sodium channel; kdr: knockdown resistance; Bl: Breteau index; WHO: World Health Organization; CDC: Center for Disease Control and Prevention.

\section{Acknowledgements}

We are grateful to Xin Liu and Yong Huang from the Shandong Institute of Parasitic Diseases for their assistance with the larval collection and insecticide usage survey.

\section{Authors' contributions}

$H L, L L, P C, L Y, J C, Y L$ and HW provided the experimental data and wrote the manuscript. XGC and MG reviewed and edited the manuscript. All authors read and approved the final manuscript.

\section{Funding}

This study was supported by grants from the National Natural Science Foundation of China [81702034 to HML, 81672059 to MQG and 81871685 to MQG], China Postdoctoral Science Foundation funded project (2017M622733 to $\mathrm{HML}$ ), and the Key Research and Development Projects in Shandong Province (2018GSF118040 to MQG).

\section{Availability of data and materials}

All data generated or analysed during this study are included in this published article and its additional files.

\section{Ethics approval and consent to participate}

Not applicable.

\section{Consent for publication}

Not applicable.

\section{Competing interests}

The authors declare that they have no competing interests.

\section{Author details}

1 Department of Pathogen Biology, Guangdong Provincial Key Laboratory of Tropical Disease Research, School of Public Health, Southern Medical University, Guangzhou, People's Republic of China. ${ }^{2}$ Shandong Institute of Parasitic Diseases, Shandong First Medical University \& Shandong Academy of Medical Sciences, Jining 272033, Shandong, People's Republic of China.

3 Jining Center for Disease Control and Prevention, Jining 272033, Shandong, People's Republic of China. ${ }^{4}$ Guangdong Provincial Institute of Biological Products and Materia Medica, Guangzhou 510440, People's Republic of China.

Received: 31 August 2019 Accepted: 1 January 2020

Published online: 09 January 2020

\section{References}

1. Gao Q, Wang F, Lv X, Cao H, Su F, Zhou J, Leng P. Aedes albopictus production in urban stormwater catch basins and manhole chambers of downtown Shanghai, China. PLoS ONE. 2018;13:e0201607.

2. Sergon K, Njuguna C, Kalani R, Ofula V, Onyango C, Konongoi LS, et al. Seroprevalence of chikungunya virus (CHIKV) infection on Lamu Island, Kenya, October 2004. Am J Trop Med Hyg. 2008;78:333-7.

3. Wei Y, Wang J, Song Z, He Y, Zheng Z, Fan P, et al. Patterns of spatial genetic structures in Aedes albopictus (Diptera: Culicidae) populations in China. Parasites Vectors. 2019;12:552.

4. Ayres CF. Identification of Zika virus vectors and implications for control. Lancet Infect Dis. 2016;16:278-9.

5. Benedict MQ, Levine RS, Hawley WA, Lounibos LP. Spread of the tiger: global risk of invasion by the mosquito Aedes albopictus. Vector Borne Zoonotic Dis. 2007;7:76-85.

6. Tian H, Huang S, Zhou S, Bi P, Yang Z, Li X, et al. Surface water areas significantly impacted 2014 dengue outbreaks in Guangzhou, China. Environ Res. 2016:150:299-305.

7. Sang S, Gu S, Bi P, Yang W, Yang Z, Xu L, et al. Predicting unprecedented dengue outbreak using imported cases and climatic factors in Guangzhou, 2014. PLoS Negl Trop Dis. 2015;9:e0003808.

8. Qin C, Shi P. Dengue in China: not a passing problem. Sci China Life Sci. 2014;57:1230-1.

9. Sun Z, Qiu X, Ren X, Wang F, Li X. Molecular tracing of pathogen of the first local dengue fever case in Hangzhou. Dis Surveill. 2017;32:132-4.

10. Chen Q, Song W, Mu D, Li Y, Yin W, Zhong L. Analysis on epidemiological characteristics of dengue fever in China, as of 31th August, 2017. Dis Surveill. 2017;32:801-4.

11. Da Cruz Ferreira DA, Degener CM, De Almeida Marques-Toledo C, Bendati MM, Fetzer LO, Teixeira CP, Eiras AE. Meteorological variables and mosquito monitoring are good predictors for infestation trends of Aedes aegypti, the vector of dengue, chikungunya and Zika. Parasites Vectors. 2017;10:78.

12. Schaffner F, Mathis A. Dengue and dengue vectors in the WHO European region: past, present, and scenarios for the future. Lancet Infect Dis. 2014:14:1271-80.

13. Sun J, Lu L, Wu H, Yang J, Xu L, Sang S, Liu Q. Epidemiological trends of dengue in mainland China, 2005-2015. Int J Infect Dis. 2017:57:86-91.

14. Wijegunawardana N, Gunawardene Y, Chandrasena T, Dassanayake RS, Udayanga N, Abeyewickreme W. Evaluation of the effects of Aedes vector indices and climatic factors on dengue incidence in Gampaha District, Sri Lanka. Biomed Res Int. 2019;2019:2950216.

15. Sanchez L, Vanlerberghe V, Alfonso L, Marquetti Mdel C, Guzman MG, Bisset J, van der Stuyft P. Aedes aegypti larval indices and risk for dengue epidemics. Emerg Infect Dis. 2006;12:800-6.

16. WHO. Dengue: guidelines for diagnosis, treatment, prevention and control: new edition. Geneva: World Health Organization; 2009. http://www. who.int/rpc/guidelines/9789241547871/en/.

17. Udayanga L, Gunathilaka N, Iabal MCM, Najim MMM, Pahalagedara K, Abeyewickreme W. Empirical optimization of risk thresholds for dengue: an approach towards entomological management of Aedes mosquitoes based on larval indices in the Kandy District of Sri Lanka. Parasites Vectors. 2018;11:368

18. Gao Q, Wang F, Lv X, Cao H, Zhou J, Su F, et al. Comparison of the humanbaited double net trap with the human landing catch for Aedes albopictus monitoring in Shanghai, China. Parasites Vectors. 2018;11:483. 
19. Wu H, Liu Q, Liu X, Lv L, Guo Y, Yu J. Surveillance for Aedes albopictus in China, 2006-2013. Dis Surveill. 2015;30:310-5.

20. Liu X, Guo Y, Wu H, Li Q, Yue Y, Ren D, et al. National surveillance on larval Aedes mosquito in China, 2015-2017. Chin J Vector Biol Control. 2018:29:325-30

21. Tun-Lin W, Kay BH, Barnes A, Forsyth S. Critical examination of Aedes aegypti indices: correlations with abundance. Am J Trop Med Hyg. 1996;54:543-7.

22. Scarnecchia DL. Mosquitoes and their control. Rangel Ecol Manag. 2004:57:684.

23. Hernandez-Triana LM, Brugman VA, Prosser SWJ, Weland C, Nikolova N, Thorne $L$, et al. Molecular approaches for blood meal analysis and species identification of mosquitoes (Insecta: Diptera: Culicidae) in rural locations in southern England, United Kingdom. Zootaxa. 2017;4250:67-76.

24. Ruiz-Arrondo I, McMahon BJ, Hernandez-Triana LM, Santibanez P, Portillo A, Oteo JA. Surveillance of mosquitoes (Diptera, Culicidae) in a northern central region of Spain: implications for the medical community. Front Vet Sci. 2019:6:86.

25. Petinaki E, Dimitracopoulos G, Spiliopoulou I. Decreased affinity of PBP3 to methicillin in a clinical isolate of Staphylococcus epidermidis with borderline resistance to methicillin and free of the mecA gene. Microb Drug Resist. 2001;7:297-300.

26. Morales Vargas RE, Phumala-Morales N, Tsunoda T, Apiwathnasorn C, Dujardin JP. The phenetic structure of Aedes albopictus. Infect Genet Evol. 2013;13:242-51.

27. Weeraratne TC, Surendran SN, Parakrama Karunaratne SHP. DNA barcoding of morphologically characterized mosquitoes belonging to the subfamily Culicinae from Sri Lanka. Parasites Vectors. 2018;11:266.

28. WHO. Monitoring and managing insecticide resistance in Aedes mosquito populations, interim guidance for entomologists. Geneva: World Health Organization; 2016. https://www.who.int/csr/resources/publications/ zika/insecticide-resistance/en/.

29. Xu J, Bonizzoni M, Zhong D, Zhou G, Cai S, Li Y, et al. Multi-country survey revealed prevalent and novel F1534S mutation in voltage-gated sodium channel (VGSC) gene in Aedes albopictus. PLoS Negl Trop Dis. 2016;10:e0004696.

30. Kasai S, Ng LC, Lam-Phua SG, Tang CS, Itokawa K, Komagata O, et al. First detection of a putative knockdown resistance gene in major mosquito vector, Aedes albopictus. Jpn J Infect Dis. 2011;64:217-21.

31. Tian Y, Wu Z, Zhang H, Wu X, Zhou M, Hong-liang C. Efficiency of three monitoring methods for the density of adult Aedes albopictus: a comparative analysis. Chin J Vector Biol Control. 2018:29:42-5.

32. Wei L, Kong Q, Hui-min W. Effectiveness of double mosquito net and BGtrap for emergency vector surveillance during dengue fever epidemics: a comparative study. Chin J Vector Biol Control. 2019;30:65-7.

33. WHO. Report of the WHO Informal Consultation on the evaluation and testing of insecticides. Geneva: World Health Organization; 1996.

34. Sanchez L, Cortinas J, Pelaez O, Gutierrez H, Concepcion D, Van der Stuyft P. Breteau index threshold levels indicating risk for dengue transmission in areas with low Aedes infestation. Trop Med Int Health. 2010;15:173-5.

35. Thammapalo S, Chongsuvivatwong V, Geater A, Dueravee M. Environmental factors and incidence of dengue fever and dengue haemorrhagic fever in an urban area, southern Thailand. Epidemiol Infect. 2008;136:135-43.

36. Luo L, Li X, Xiao X, Xu Y, Huang M, Yang Z. Identification of Aedes albopictus larval index thresholds in the transmission of dengue in Guangzhou, China. J Vector Ecol. 2015;40:240-6.

37. Tangena JA, Thammavong P, Hiscox A, Lindsay SW, Brey PT. The human-baited double net trap. An alternative to human landing catches for collecting outdoor biting mosquitoes in Lao PDR. PLOS ONE. 2015;10:e0138735.
38. Chi J, Gu W, Chen W, Lei Y. Study on the correlation of three monitoring methods and application in epidemic area of dengue fever. Chin J Vector Biol Control. 2015:26:495-7.

39. Xiang J, Hansen A, Liu Q, Liu X, Tong MX, Sun Y, et al. Association between dengue fever incidence and meteorological factors in Guangzhou, China, 2005-2014. Environ Res. 2017;153:17-26.

40. LiuT, Zhu G, He J, Song T, Zhang M, Lin H, et al. Early rigorous control interventions can largely reduce dengue outbreak magnitude: experience from Chaozhou, China. BMC Public Health. 2017;18:90.

41. Guo YH, Lai SJ, Liu XB, Li GC, Yu HJ, Liu QY. Governmental supervision and rapid detection on dengue vectors: an important role for dengue control in China. Acta Trop. 2016;156:17-21.

42. Gao JP, Chen HM, Shi H, Peng H, Ma YJ. Correlation between adult pyrethroid resistance and knockdown resistance $(k d r)$ mutations in Aedes albopictus (Diptera: Culicidae) field populations in China. Infect Dis Poverty. 2018;7:86.

43. Wang Y, Yu W, Shi H, Yang Z, Xu J, Ma Y. Historical survey of the kdr mutations in the populations of Anopheles sinensis in China in 1996-2014. Malar J. 2015;14:120.

44. Li Y, Xu J, Zhong D, Zhang H, Yang W, Zhou G, et al. Evidence for multipleinsecticide resistance in urban Aedes albopictus populations in southern China. Parasites Vectors. 2018;11:4.

45. Auteri M, La Russa F, Blanda V, Torina A. Insecticide resistance associated with kdr mutations in Aedes albopictus: an update on worldwide evidences. Biomed Res Int. 2018;2018:3098575.

46. Smith LB, Kasai S, Scott JG. Pyrethroid resistance in Aedes aegypti and Aedes albopictus: important mosquito vectors of human diseases. Pestic Biochem Physiol. 2016;133:1-12.

47. Kasai S, Caputo B, Tsunoda T, Cuong TC, Maekawa Y, Lam-Phua SG, et al. First detection of a Vssc allele V1016G conferring a high level of insecticide resistance in Aedes albopictus collected from Europe (Italy) and Asia (Vietnam), 2016: a new emerging threat to controlling arboviral diseases. Euro Surveill. 2019:24:1700847.

48. Stenhouse SA, Plernsub S, Yanola J, Lumjuan N, Dantrakool A, Choochote W, Somboon P. Detection of the V1016G mutation in the voltage-gated sodium channel gene of Aedes aegypti (Diptera: Culicidae) by allele-specific PCR assay, and its distribution and effect on deltamethrin resistance in Thailand. Parasites Vectors. 2013;6:253.

49. Li CX, Kaufman PE, Xue RD, Zhao MH, Wang G, Yan T, et al. Relationship between insecticide resistance and $k d r$ mutations in the dengue vector Aedes aegypti in southern China. Parasites Vectors. 2015;8:325.

50. Sang $\mathrm{S}$, Chen $\mathrm{B}, \mathrm{Wu} \mathrm{H}$, Yang Z, Di B, Wang L, et al. Dengue is still an imported disease in China: a case study in Guangzhou. Infect Genet Evol. 2015:32:178-90.

51. Qi-yong L. Epidemic profile of vector-borne diseases ABD vector control strategies in the new era. Chin JVector Biol Control. 2019;30:1-6.

\section{Publisher's Note}

Springer Nature remains neutral with regard to jurisdictional claims in published maps and institutional affiliations.

Ready to submit your research? Choose BMC and benefit from:

- fast, convenient online submission

- thorough peer review by experienced researchers in your field

- rapid publication on acceptance

- support for research data, including large and complex data types

- gold Open Access which fosters wider collaboration and increased citations

- maximum visibility for your research: over 100M website views per year

At $\mathrm{BMC}$, research is always in progress.

Learn more biomedcentral.com/submissions 